\title{
Two-dimensional scattering and bound states of polar molecules in bilayers
}

\author{
Michael Klawunn ${ }^{1,2}$, Alexander Pikovski ${ }^{1}$ and Luis Santos ${ }^{1}$ \\ ${ }^{1}$ Institut für Theoretische Physik, Leibniz Universität Hannover, Appelstr. 2, 30167 Hannover, Germany \\ ${ }^{2}$ INO-CNR BEC Center and Dipartimento di Fisica, Università di Trento, 38123 Povo, Italy
}

(Dated: May 29, 2022)

\begin{abstract}
Low-energy two-dimensional scattering is particularly sensitive to the existence and the properties of weakly bound states. We show that interaction potentials $V(r)$ with vanishing zero-momentum Born approximation $\int d r r V(r)=0$ lead to an anomalously weak bound state which crucially modifies the two-dimensional scattering properties. This anomalous case is especially relevant in the context of polar molecules in bilayer arrangements.
\end{abstract}

PACS numbers: $34.50 . \mathrm{Cx}, 67.85 .-\mathrm{d}$

\section{INTRODUCTION}

Ultracold atomic gases are many-body systems, but most of their fundamental properties originate from the underlying two-body problem, given by an interaction potential which is typically considered as short-range. The situation is completely different in dipolar gases due to the long-range character of the dipole-dipole interaction [1, 2]. Polar molecules with a potentially large electric dipole moment constitute a particularly exciting dipolar gas. However, achieving quantum degeneracy is handicapped by exothermic chemical reactions [3]. The latter may be avoided by confining the gas in twodimensional geometries if the dipoles are polarized perpendicular to the trap plane, due to the repulsive character of the dipole-dipole interaction [4].

Bilayer (and in general multi-layer) arrangements of polar molecules offer the possibility of stability against inelastic reactions and give rise to inter-layer pairing due the dipole-dipole force [5-7]. A dipole in layer 1 interacts with a dipole in layer 2 , both dipoles oriented perpendicularly to the layers, by the potential

$$
V_{\mathrm{dd}}(r)=U_{0}\left(r^{2}-2\right) /\left(r^{2}+1\right)^{5 / 2} .
$$

Here $r$ is the relative in-plane distance between the two dipoles, the inter-layer distance $\lambda$ is set to 1 , and $U_{0}$ is a positive dimensionless coupling constant. We have $U_{0}=m d^{2} / \hbar^{2} \lambda$ and the unit of energy is $E_{0}=\hbar^{2} / m \lambda^{2}$, with $m$ the mass and $d$ dipole moment of the molecule. This potential is attractive at short distances and repulsive at large distances, fulfilling the peculiar condition $\int d r r V_{\mathrm{dd}}(r)=0$, i.e. its zero-momentum Born approximation vanishes [7 9]. Inter-layer interactions of a different type may be attained in binary mixtures, where one of the species is confined in a bilayer while the other moves freely. The free species mediates a $2 \mathrm{D}$ interaction with a RKKY-like potential [10], which may as well have a vanishing zero-momentum Born approximation.

Low energy 2D scattering, which determines the properties of 2D quantum gases [11 13], is particularly sensitive to the existence and properties of weakly bound states. Although 2D scattering [14-18] and weakly bound states [19 21] have been intensively studied, little is known for the case when $\int \operatorname{drr} V(r)=0$. The binding energy for weakly coupled bound states in this case was calculated in Ref [19]. However, a detailed investigation of the binding energy at larger coupling and of the lowenergy scattering properties is still lacking.

In this Brief Report we discuss the low-energy scattering and weakly bound states for radial potentials $V(r)$ satisfying $\int d r r V(r)=0$. We extend (using an alternative method) the expression derived in Ref. [19] for the binding energy of the weakly bound state. We show that the presence of this anomalously weak bound state modifies significantly the scattering amplitude compared to the usual case of potentials with non-vanishing zeromomentum Born approximation. As an example, we specialize for the potential $V_{\mathrm{dd}}$ appearing in bilayer gases of polar molecules and check the validity of the obtained analytical expressions using exact numerical calculations.

This paper is organized as follows. In Sec. III, we introduce the Jost function formalism to study the binding energy of weakly bound states and evaluate the general expressions for $V_{\mathrm{dd}}$. In Sec. III we discuss the modifications introduced in the two-dimensional scattering properties if $\int d r r V(r)=0$ and study this for $V_{\mathrm{dd}}$ in detail. Our conclusions are summarized in Sec. IV.

\section{WEAKLY BOUND STATES}

Ref. 19] studied the bound states of the Schrödinger equation $\left\{-\nabla^{2}+V(\mathbf{r})\right\} \psi=\epsilon \psi$ for potentials of the form $V(\mathbf{r})=U_{0} v(\mathbf{r})$, where as above $U_{0}$ denotes a positive dimensionless coupling constant characterizing the potential strength [26]. For the case of weak coupling $\left(U_{0} \rightarrow 0\right)$ it was shown that a shallow bound state always exists if $\int d^{2} r v(\mathbf{r}) \leq 0$, but there is no bound state if $\int d^{2} r v(\mathbf{r})>0$. Furthermore, it was shown that for $\int d^{2} r v(\mathbf{r})<0$ the binding energy of the shallow bound state is of the form $\epsilon_{b} \sim-\exp \left[4 \pi / \int d^{2} r V(\mathbf{r})\right]$, as expected from e.g. Ref. [22]. However, for our case of interest $\int d^{2} r v(\mathbf{r})=0$, the binding energy acquires the anomalous form $\epsilon_{b} \sim-\exp \left[1 / c U_{0}^{2}\right]$, with

$$
c=\frac{1}{8 \pi} \int d^{2} r \int d^{2} r^{\prime} v(\mathbf{r}) \ln \left|\mathbf{r}-\mathbf{r}^{\prime}\right| v\left(\mathbf{r}^{\prime}\right) .
$$


It can be shown that $c<0$ for any $v(\mathbf{r})$. For the potential $V_{\mathrm{dd}}(r)$ appearing in a bilayer system of polar molecules, one obtains $c=-1 / 8$, and hence the binding energy becomes:

$$
\epsilon_{b}^{\mathrm{dd}} \sim-\exp \left\{-8 / U_{0}^{2}\right\} .
$$

However, a numerical calculation of the binding energy (see below) shows that this result is not very accurate even for very small $U_{0}$. This motivates us to derive a more accurate analytic expression for the binding energy which remains valid for larger $U_{0}$.

The two-body scattering problem for a radially symmetric potential $V(r)$ in two dimensions is described by the Schrödinger equation

$$
\left\{-\left(\frac{d^{2}}{d r^{2}}+\frac{1}{r} \frac{d}{d r}\right)+V(r)\right\} \phi(r)=k^{2} \phi(r),
$$

where all quantities are dimensionless and $\phi(r)$ is the radial wavefunction. Only $s$-waves are considered since we are only interested in low-energy properties. Following Ref. [23], we employ the Jost function formalism to study the scattering problem and the shallow bound states. The definition of the Jost function $\mathcal{F}(k)$ in the 2D case may be found in Ref. [23]. The properties of $\mathcal{F}(k)$ are similar to those of the Jost function for the 3D case 24].

The scattering phase shift $\delta(k)$ is related to $\mathcal{F}(k)$ by

$$
\tan \delta(k)=-\frac{\operatorname{Im} \mathcal{F}(k)}{\operatorname{Re} \mathcal{F}(k)},
$$

the scattering amplitude is

$$
f(k)=\frac{\tan \delta(k)}{1-i \tan \delta(k)},
$$

and $\sigma=(4 / k)|f(k)|^{2}=(4 / k) \sin ^{2} \delta(k)$ is the total 2D swave cross-section. For complex $k$, the zeros of the Jost function on the positive imaginary axis, $\mathcal{F}(i \alpha)=0$ with $\alpha>0$, are the bound states of the potential with binding energy $\epsilon_{b}=-\alpha^{2}$.

The following integral representation of the Jost function $\mathcal{F}(k)$ will be employed below:

$$
\mathcal{F}(k)=1+e^{i \pi / 4} \sqrt{\frac{\pi}{2 k}} \int_{0}^{\infty} d r \sqrt{r} V(r) J_{0}(k r) f_{0}(k r),
$$

where $f_{0}(k, r)$ satisfies the integral equation

$$
f_{0}(k, r)=\sqrt{\frac{i \pi k r}{2}} H_{0}(k r)+\int_{r}^{\infty} d s g(k, r, s) V(s) f_{0}(k, s),
$$

with

$$
g\left(k, r, r^{\prime}\right)=\frac{\pi}{2} \sqrt{r r^{\prime}}\left[J_{0}(k r) Y_{0}\left(k r^{\prime}\right)-J_{0}\left(k r^{\prime}\right) Y_{0}(k r)\right] .
$$

In the previous expressions $J_{0}, Y_{0}$ are Bessel functions, and $H_{0}$ is the Hankel function of the first kind.

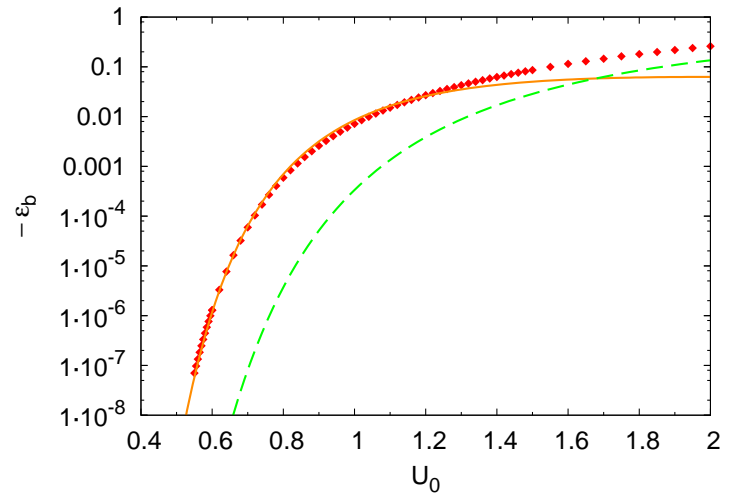

FIG. 1: (Color online) Binding energy for $V_{\mathrm{dd}}$ as a function of the dipole strength $U_{0}$ calculated numerically (dots), from equations (16) (solid) and (3) (dashed).

In the following we are interested in determining the weakly bound states. To this aim we expand the Jost function for small $k$ :

$$
\mathcal{F}(k)=A \ln k+B-i \frac{\pi}{2} A,
$$

where $A, B$ are real constants independent of $k$. These constants can be represented by infinite series of the form $A=\sum A_{j}, B=1-\sum B_{j}[27]$. The first terms of the $A_{j}$ series are of the form:

$$
\begin{aligned}
& A_{1}=-\int_{0}^{\infty} d r r V(r) \\
& A_{2}=-\int_{0}^{\infty} d r r V(r) \int_{r}^{\infty} d s s V(s) \ln \left(\frac{s}{r}\right),
\end{aligned}
$$

whereas those of the $B_{j}$ series are:

$$
\begin{aligned}
& B_{1}=\int_{0}^{\infty} d r r V(r) \ln (r)-C_{1} \\
& B_{2}=\int_{0}^{\infty} d r r V(r) \int_{r}^{\infty} d s s V(s) \ln \left(\frac{s}{r}\right) \ln (s)-C_{2},
\end{aligned}
$$

with $C_{i}=A_{i} \ln \left(e^{\gamma} / 2\right)$ and $\gamma \approx 0.577$ the Euler constant.

As mentioned above, the bound states are given by the zeros of $\mathcal{F}(k)$ on the positive imaginary axis. Using (9) with $k=i \alpha$ we hence obtain the expression of the binding energy:

$$
\epsilon_{b}=-\exp \left\{-2 \frac{B}{A}\right\} .
$$

Note that Eq. (14) is valid as long as the binding energy is small enough such that the logarithmic term dominates the Jost function. For potentials with $\int d r r V(r)<0$, it is sufficient to take $A \approx A_{1}$ and $B \approx 1$, recovering the expression $\epsilon_{b} \sim \exp \left(4 \pi / \int d^{2} r V(r)\right)$ for small $U_{0}$.

However, for the case of potentials such that $\int \operatorname{drr} V(r)=0$, we have $A_{1}=0$, and the first nonvanishing term is $A \approx A_{2}, B \approx 1$, providing an alternative derivation of Eq. (3). A more precise formula is 


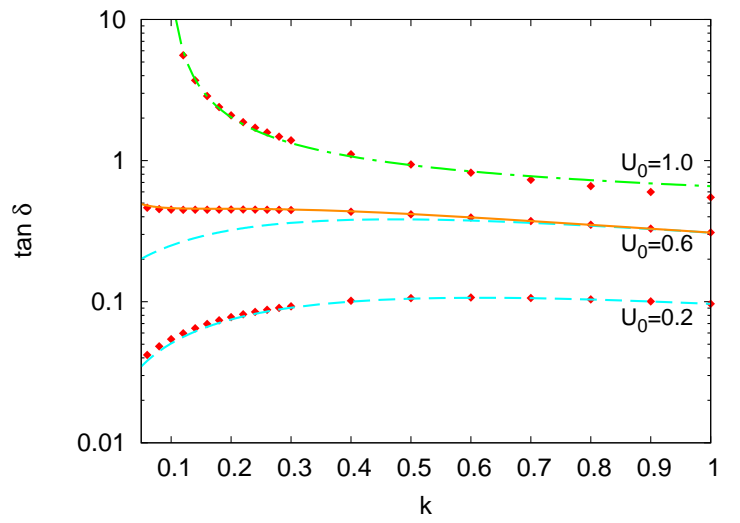

FIG. 2: (Color online) Scattering phase shift for $V_{\mathrm{dd}}$ as a function of $k$ for different $U_{0}$ calculated numerically (dots) compared to the log-behavior (20) for $U_{0}=1.0$ (dash-dotted, green), to formula (17) for $U_{0}=0.6$ (solid, orange) and to the second Born approximation (21) for $U_{0}=0.6$ and $U_{0}=0.2$ (dashed, cyan).

obtained by including higher-order terms:

$$
\epsilon_{b}=-\exp \left\{-2 \frac{1-B_{1}-B_{2}-\ldots}{A_{1}+A_{2}+\ldots}\right\} .
$$

For the case of the inter-layer dipole-dipole potential $V_{\mathrm{dd}}$, the integrals can be carried out analytically to find a corrected expression for the binding energy

$$
\epsilon_{b}^{\mathrm{dd}} \simeq-\exp \left\{-\frac{8}{U_{0}^{2}}\left[1-U_{0}+\frac{U_{0}^{2}}{4}\left(\frac{5}{2}+\ln \frac{e^{\gamma}}{2}\right)\right]\right\} .
$$

Figure 1 compares the numerical result for the binding energy for $V_{\mathrm{dd}}$ (obtained directly from the 2D Schrödinger equation) with the analytical expressions of Eq. (3) and (16). Note that whereas Eq. (3) provides a relative inaccurate approximation even at rather low $U_{0}$, the newly derived expression (16) is in excellent agreement with the numerics, all the way to $U_{0} \lesssim 1.2$.

Finally, we note that for large $U_{0}$ the binding energy for $V_{\mathrm{dd}}$ can be determined by a variational calculation, giving [25] $\epsilon_{b}^{\mathrm{dd}} \approx-2 U_{0}+4 \sqrt{3 U_{0} / 2}-15 / 4$, which coincides with the numerics only for $U_{0} \gtrsim 5$.

\section{SCATTERING PHASE SHIFT}

We have shown above that the Jost function formalism is particularly useful for the analysis of weakly bound states. In this section we employ this formalism for the study of $2 \mathrm{D}$ scattering and in particular for the calculation of the $s$-wave scattering phase shift $\delta(k)$.

An approximate expression of the Jost function $\mathcal{F}(k)$ for small $U_{0}$ is obtained by iterating twice the integral equation (8). Note that we keep all orders in $k$. The resulting scattering phase shift follows from the relation (5):

$$
\tan \delta(k)=\frac{-\frac{\pi}{2} \mathrm{I}_{J J}(k)-\frac{\pi^{2}}{4}\left(\mathrm{I}_{J J, J Y}(k)-\mathrm{I}_{J Y, J J}(k)\right)}{1-\frac{\pi}{2} \mathrm{I}_{J Y}(k)-\frac{\pi^{2}}{4}\left(\mathrm{I}_{J J, Y Y}(k)-\frac{1}{2} \mathrm{I}_{J Y}^{2}(k)\right)},
$$

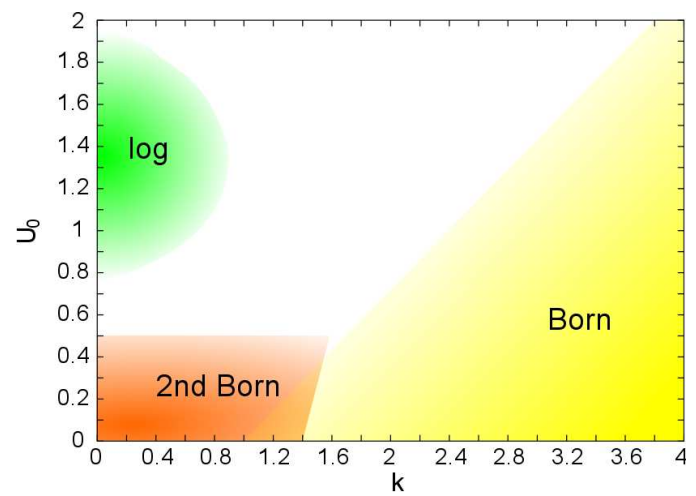

FIG. 3: (Color online) Qualitative sketch of the regimes of $k$ and $U_{0}$ where the scattering phase shift for $V_{\mathrm{dd}}$ can be approximated by the logarithm Eq. (20) (green), the Born approximation Eq. (22) (yellow), and the second Born approximation Eq. (21) (orange).

where we have introduced the notation

$$
\begin{aligned}
& I_{F G}=\int_{0}^{\infty} d r r V(r) F(r) G(r), \\
& I_{F G, P Q}=\int_{0}^{\infty} d r r V(r) F(r) G(r) \int_{r}^{\infty} d s s V(s) P(s) Q(s),
\end{aligned}
$$

and $J, Y$ stand for $J_{0}(k r)$ and $Y_{0}(k r)$.

For small $k$ it is possible to simplify Eq. (17). Employing the logarithmic expression (91), the relation (5), and the expression for the binding energy (14), we recover the well known logarithmic expression (see e.g. [12])

$$
\tan \delta(k)=\left[\frac{1}{\pi} \ln \frac{k^{2}}{\left|\epsilon_{b}\right|}\right]^{-1}
$$

characteristic of 2D scattering, which relates the scattering shift and the binding energy of the weakly bound state. However, for the case $\int \operatorname{drr} V(r)=0$ the binding energy $\left|\epsilon_{b}\right|$ can become anomalously small, and hence the expression at the right-hand side of Eq. (20) can become very small for reasonable $k$. In this case, it is not any more the leading term for the low-energy scattering.

On the other hand, for large enough $k$ and small $U_{0}$, the first integral $\mathrm{I}_{J J}(k)$ in Eq. (17) dominates, and we recover, as expected for sufficiently large $k$, the Born approximation. Therefore, formula (17) interpolates smoothly between the correct low-energy and the correct high-energy behavior. This suggests that it may be valid, at least qualitatively, even for large $U_{0}$. By expanding Eq. (17) in powers of $U_{0}$, one recovers the Born series, which reads to second order:

$$
\tan \delta(k)=-\frac{\pi}{2} I_{J J}-\frac{\pi^{2}}{2} I_{J J, J Y}
$$

It is interesting to discuss these results for the case of the interlayer dipole-dipole potential $V_{\mathrm{dd}}$. We have computed the scattering phase-shift numerically from the Schrödinger equation and compared it to the results of Eq. (17), obtaining that Eq. (17) provides the correct 
scattering phase-shift with excellent accuracy, at least in the range $0.03 \leq k \leq 5$ and $0.05 \leq U_{0} \leq 2.0$. Hence Eq. (17) is a good approximation not only for $U_{0} \ll 1$, but also for $U_{0} \sim 1$. Of course, if the interactions are too large $\left(U_{0} \gg 1\right)$, the contributions from higher iterations of the integral equation (8) become more important and Eq. (17) looses its accuracy.

Figure 2 compares the numerical results for the scattering phase-shift for $V_{\mathrm{dd}}$ with the limiting cases provided by expressions (20) and (21) for small $k$ and different values of $U_{0}$. It is seen that for $U_{0} \sim 1$, the scattering phase-shift is best approximated by the logarithmic expression (20), and for $U_{0} \ll 1$ by the second Born approximation (21). For intermediate values of $U_{0}$ none of the limiting cases is accurate and the full expression (17) must be used. We sketch in Fig. 3 qualitatively the regimes of $k$ and $U_{0}$, where the logarithm (20), the first Born approximation (22), and the second Born approximation (21) are good approximations, as obtained by comparison with the numerical solution. Note that, excluding unreasonably small $k$, the logarithmic form (20) is just valid for $k<1$ and the window $0.7 \lesssim U_{0} \lesssim 2.0$.

Finally, we note that the first Born approximation for $V_{\text {dd }}$ can be evaluated exactly analytically:

$$
\tan \delta^{\mathrm{dd}}(k) \simeq-\frac{\pi}{2} U_{0}\left[-\frac{4 k}{\pi}-2 k\left(\mathbf{L}_{1}(2 k)-I_{1}(2 k)\right)\right]
$$

where $\mathbf{L}_{1}$ is the modified Struve function. The second Born approximation (21) can be expanded for small $k$

$$
\tan \delta^{\mathrm{dd}}(k) \approx 2 U_{0} k-\pi U_{0} k^{2}+\frac{1}{8} U_{0}^{2} \pm \ldots,
$$

which gives a maximum in the scattering phase shift at $k \approx 1 / \pi$ as observed in the numerical results.

\section{CONCLUSIONS}

In conclusion, two-dimensional radial interaction potentials $V(r)$ with a vanishing zero-momentum Born approximation, $\int d r r V(r)=0$, result in interesting physics crucially different from purely attractive or purely repulsive potentials. Using the Jost function formalism, we have derived an expression for the binding energy as a function of the potential strength $U_{0}$, which remains accurate for a wide regime of $U_{0}$ values. Moreover, we have investigated the scattering amplitude in different parameter regimes. In particular, we have shown a significant deviation of the scattering behavior in comparison with potentials with $\int d r r V(r) \neq 0$, due to the anomalously low binding energy of the weakly bound state.

These results are of particular importance in the physics of two-dimensional systems, and more specifically on two-dimensional ultracold gases. Standard theories, in particular the theory of BCS-BEC crossover [12, 13], are based on the fact that the scattering amplitude possesses the logarithmic dependence (20). These results are therefore modified if the potential has a vanishing zero-momentum Born approximation. This has particularly important consequences for the properties of a gas of polar Fermi molecules confined in a bilayer geometry, including inter-layer pairing [7].

\section{Acknowledgments}

We thank A. Recati and G.V. Shlyapnikov for helpful discussions. We acknowledge the support of the DFG (QUEST Cluster of Excellence).
[1] M. A. Baranov, Phys. Rep. 464, 71 (2008).

[2] T. Lahaye, C. Menotti, L. Santos, M. Lewenstein and T. Pfau, Rep. Prog. Phys. 72, 126401 (2009).

[3] S. Ospelkaus et al., Science 327, 853 (2010).

[4] K.-K. Ni et al., Nature 464, 1324 (2010).

[5] M. Klawunn, J. Duhme, and L. Santos, Phys. Rev. A 81, 013604 (2010).

[6] A. C. Potter, E. Berg, D.-W. Wang, B. I. Halperin and E. Demler, arXiv:1007.5061

[7] A. Pikovski, M. Klawunn, G. V. Shlyapnikov and L. Santos, arXiv:1008.3264.

[8] S.-M. Shih and D.-W. Wang, Phys. Rev. A 79, 065603 (2009).

[9] J. R. Armstrong et al., EPL 91, 16001 (2010).

[10] Y. Nishida, Phys. Rev. A 82, 011605 (2010).

[11] D. S. Petrov, M. A. Baranov and G. V. Shlyapnikov, Phys. Rev. A 67, 031601(R) (2003).

[12] M. Randeria, J.-M. Duan and L.-Y. Shieh, Phys. Rev. B 41, 327 (1990);

[13] K. Miyake, Prog. Theo. Phys. 69, 1794 (1983).

[14] D. Bollé and F. Gesztesy, Phys. Rev. Lett. 52, 1469 (1984).
[15] D. Bollé and F. Gesztesy, Phys. Rev. A 30, 1279 (1984).

[16] W.G. Gibson, Phys. Lett. A 117, 107 (1986).

[17] N. N. Khuri, A. Martin, J.-M. Richard and T. T. Wu, J. Math. Phys. 50, 072105 (2009).

[18] K. Chadan, N. N. Khuri, A. Martin and T. T. Wu, Phys. Rev. D 58, 025014 (1998).

[19] B. Simon, Annals of Physics 97, 279 (1976).

[20] S. H. Patil, Phys. Rev. A 22, 2400 (1980).

[21] S. H. Patil, Phys. Rev. A 25, 2467 (1982).

[22] L. D. Landau and E. M. Lifshitz, Quantum Mechanics, Butterworth-Heinemann, Oxford, 1977.

[23] R. G. Newton, J. Math. Phys. 27, 2720 (1986).

[24] R. G. Newton, Scattering theory of waves and particles, New York, McGraw-Hill, 1966.

[25] V. I. Yudson, M. G. Rozman, and P. Reineker, Phys. Rev. B 55, 5214 (1997).

[26] In Ref. 19] it was assumed that $\int d^{2} r\left(1+|\mathbf{r}|^{\delta}\right)|v(\mathbf{r})|<\infty$, with $\delta>0$.

[27] We always assume $A \neq 0$. The exceptional case $A=0$ (cf. 23]) is the two-dimensional analogue of zero-energy resonance in three dimensions. 